\title{
INFLUÊNCIA DE VARIÁVEIS METEOROLÓGICAS SOBRE A INCIDÊNCIA DO DENGUE, MENINGITE E PNEUMÔNIA EM JOÃO PESSOA-PB
}

\author{
NADJA MARIA NASCIMENTO SOUSA ${ }^{1}$, RENÍLSON TARGINO DANTAS ${ }^{2}$ e \\ RODRIGO CÉZAR LIMEIRA ${ }^{3}$
}

\author{
1, 2, 3 Universidade Federal de Campina Grande, Unidade Acadêmica de Ciências Atmosféricas, Bloco CL. \\ Avenida Aprígio Veloso, 882, Tel.: (83) 3101202, Bodocongó, Campina Grande, PB, CEP: 58109-970. \\ E-mails: ${ }^{2}$ renilson@dca.ufcg.edu.br; ${ }^{3}$ rodrigocezarlimeira@yahoo.com.br
}

Recebido Março 2005 - Aceito Setembro 2006

\begin{abstract}
RESUMO
Foram utilizados dados de temperatura, umidade relativa do ar e precipitação do período de 1992 a 2000, de João Pessoa $\left(07^{\circ} 07^{\prime} \mathrm{S} ; 034^{\circ} 53 \mathrm{~W} ; 05 \mathrm{~m}\right)$ objetivando identificar a influência destes elementos meteorológicos na incidência do dengue, meningite e pneumonia. Determinaram-se os coeficientes de incidência mensal por 10.000 habitantes, relacionando-os com as temperaturas médias (T), máximas (Tx), mínimas (Tn), umidade relativa do ar (UR) e precipitação (P) do período estudado. Utilizou-se a precipitação, em totais mensais, e as temperaturas, mínima, média e máxima e umidade relativa do ar em médias mensais.Utilizaram-se as variações climáticas mensais e por estações do ano no período estudado. Fez-se análise de regressão e a regressão linear múltipla dos elementos meteorológicos do modelo, para saber, o máximo que estes explicam sobre a incidência das patologias estudadas.Utilizouse o método de regressão múltipla, "stepwise", mantendo no modelo os elementos meteorológicos que mais contribuíam sobre a incidência das doenças, teste de hipóteses, F, com nível de significância de $5 \%$. Houve influência dos elementos meteorológicos na incidência do Dengue, meningite e pneumonia em João Pessoa com $\mathrm{R}^{2}$ de $0,34,0,25$ e 0,23 respectivamente. Os elementos meteorológicos preditores da incidência das doenças em João Pessoa, são a Temperatura máxima e a Precipitação.
\end{abstract}

Palavras - chave: correlação, incidência, epidemia.

\begin{abstract}
INFLUENCE OF THE METEOROLOGICAL VARIABLES ON THE INCIDENCE OF PRIMNESS, MENINGITE AND PNEUMONIA AT JOÃO PESSOA-PB

It was used of temperature data and relative humidity of the air and precipitation to the period from 1992 to 2000, of João Pessoa $\left(07^{\circ} 07^{\prime} \mathrm{S}\right.$; 34 53 W;05m) aiming at identifying the influence of these meteorological elements in the incidence of Primness, meningitis and pneumonia. Initially, it determined the coefficient of monthly incidence goes 10.000 in habitants, relating them with: average temperature $(T)$, maximum $\left(T_{x}\right)$, minimum $\left(T_{n}\right)$, relative humidity (UR) and precipitation (P). It was used the precipitation, in total monthly, temperatures, low, average and maximum and relative humidity was used in monthly averages. The climatic variations were observed, monthly and go seasons. It was made analysis of simple regression and, the multiple lineal regression with the meteorological elements in the model, goes to know the maximum that these explain on the incidence of pathologies. Was made it uses of the method of multiple regression" stepwise ". The test of hypotheses, F, with level of the significance of $5 \%$ was influence of the meteorological elements in the incidence of primness, meningitis and pneumonia in João Pessoa with R2 0,34, 0,25 and 0,23, respectably. The meteorological elements predictors to incidence of the disease in João Pessoa are maximum temperature and precipitation.
\end{abstract}

Keywords: correlation, incidence, epidemic. 


\section{INTRODUÇÃO}

O Brasil, país tropical, onde a maioria da população é desnutrida e vive em condições sanitárias precárias, o aparecimento de doenças do trato respiratório e endêmico, ocorrem freqüentemente. Estes fatores associados às mudanças climáticas contribuem para o aumento de algumas destas patologias. Doenças transmitidas por vetores (como dengue) e por outros organismos patogênicos, são um grave problema no processo saúde - doença.

Dengue, é uma patologia causada por um vírus que aparece nos trópicos, transmitida por mosquitos da espécie Aedes aegypti. A re-emergência dessa infecção sob a forma de Dengue Clássico, Febre Hemorrágica do Dengue e Síndrome de Choque do Dengue coloca essa virose como um dos mais graves problemas de Saúde Pública do continente. No Brasil, a primeira epidemia ocorreu em 1982, em Boa Vista, Roraima. Somente a partir de 1986, se iniciou, como epidemias explosivas, que foram se expandindo para todas as regiões brasileiras. Nesse momento, o Brasil alcançou um elevado índice endêmico, em virtude da rápida dispersão do vetor em grande extensão territorial, o que propiciou a circulação viral em maior número de estados e municípios, expondo, paulatinamente, novas populações às infecções.

Meningite é uma infecção do sistema nervoso central, com acometimento das meninges, causado por múltiplas etiologias e caracterizada por febre, cefaléia intensa, vômitos, sinais de irritação meníngea e alteração do líquido cefáloraquidiano.

Quanto ao modo de transmissão, variam de acordo com o agente infeccioso, podendo ser de pessoa para pessoa, através das gotículas de secreções da nasofaringe, meningite por Haemophilus influenzae, por via aérea, meningite tuberculosa, e de acordo com o agente etiológico, meninges virais.

Pneumonia, refere-se a infecção do trato respiratório inferior, primariamente envolve o pulmão, e constitui a principal causa infecciosa de atendimento médico, sendo responsável por um dos maiores percentuais de receitas de antibióticos. No Brasil, as pneumonias são a primeira causa de morte entre as doenças respiratórias.

De acordo com Rouquayrol (1994), no campo epidemiológico, o tempo é o aspecto do ambiente físico que tem até agora concentrado maior atenção para estudos epidemiológicos. O clima é a resultante de toda variedade de fenômenos meteorológicos específicos, que caracterizam a situação média da atmosfera, em uma região delimitada da superfície terrestre. Estudam-se os fatores climáticos, para que, através deles, possam ser inferidas hipóteses de causalidade quanto a algum fator de risco cuja variação na natureza dependa da variação de algum fator climático.
Segundo a mesma autora, os aspectos do clima que mais de perto influenciam as biocenoses e, portanto, os seres vivos implicados no processo de transmissão de doença são a temperatura, a umidade relativa do ar e a precipitação pluviométrica.

É de grande importância para a biometeorologia animal, fazer estudos relacionando clima e o tempo com a saúde. Esta é uma de crescente preocupação internacional por parte de médicos e climatologistas, à medida que aparecem mais evidências de que um aquecimento global do planeta possa aumentar as possibilidades de que vetores ampliem suas áreas de influência ao propagarem vírus e outros microorganismos. Além do mais, anos com ocorrência de eventos extremos, como El Niño, por exemplo, aparecem relacionados com eclosão de doenças transmitidas, principalmente, por vetores como dengue e também ocorre um aumento considerável de doenças respiratórias. Justifica-se dessa forma, a importância de fazer-se um estudo a respeito da dengue, oportunizando a comunidade acadêmica e a sociedade, informações de como a mudança de tempo influencia sobre a mesma.

Nesta perspectiva, este trabalho tem como objetivo conduzir tecnicamente um estudo sobre a influência dos elementos meteorológicos sobre a incidência, mensal e por estação do ano, da dengue, meningite e pneumonia na cidade de João Pessoa-PB.

\section{MATERIAL E MÉTODOS}

Foram utilizados dados de temperatura, umidade relativa do ar e precipitação referentes ao período de 1992 a 2000 de João Pessoa-PB cujas coordenadas geográficas são: (0707 `S; $34^{\circ} 53^{\prime} \mathrm{W}$; 05m), para identificar a influência destes elementos meteorológicos sobre a incidência do dengue, meningite e pneumonia em João Pessoa no Estado da Paraíba, localidade de clima Tropical Úmido, com chuvas em grande quantidade, praticamente o ano todo.

Para verificar o aumento (ou redução) da ocorrência das doenças, fez-se uso do conceito de incidência, que é utilizado para efeito de relativização da medida, e de sua aplicação ao estudo da incidência de doenças, numa população em épocas diferentes, ou em populações diversas numa mesma época.

Os coeficientes de incidência mensal $\left(\mathrm{C} \cdot \mathrm{I}_{\mathrm{t}}\right)$ para cada doença, para o período de 1992 - 2000, foram calculados da seguinte forma Rouquayrol (1994):

$\mathrm{C} \cdot \mathrm{I}_{\mathrm{t}}=\frac{\text { número de casos novos } \times 10^{\mathrm{n}}}{\text { população exposta ao risco }}$

Em que n, determina o tamanho da amostra da população e t, um mês do ano. Neste trabalho, o valor de $n$ é igual a 4, e portanto, os coeficientes de incidências das patologias, foram expressos por 10.000 habitantes. 
O coeficiente de incidência mensal médio esperado para cada mês dos anos posteriores a 2000, foi dado por:

$C \cdot$ Iméd $_{{ }_{t}}=Y_{t}$

onde $Y_{t}$ é a média aritmética dos coeficientes de incidência calculados para todos os meses equivalentes, no período estudado.

Já, o coeficiente de incidência mensal máximo esperado, para cada mês do ano, foi dado por:

$\mathrm{C} \cdot \operatorname{Imáx}_{\mathrm{t}}=\mathrm{C} \cdot$ Iméd $_{\mathrm{t}}+\mathrm{ZS} \mathrm{S}_{\mathrm{t}}$

onde z, é o número de desvios padrões que uma variável aleatória está afastada da média; e $\mathrm{S}_{\mathrm{t}}$ é o desvio - padrão, dado por:

$S_{t}=\left(\sum_{i=1}^{n}\left(Y_{i}-\bar{Y}\right)^{2} / n-1\right)^{1 / 2}$

em que $\mathrm{Y}$, representa uma patologia, té um mês do ano e n, o tamanho da amostra, na referida equação.

Neste trabalho, foi assumido o valor de 1,64 para $\mathrm{z}$, quando se referir ao cálculo do C·I. máx $\mathrm{t}$ e z igual a 1,96, para o intervalo de confiança dos dados meteorológicos (média aritmética do referido elemento $\pm 1,96 \mathrm{~S}$ ), conforme Bussab (1998).

Para os dados referentes às patologias, adquiridos junto a Fundação Nacional de Saúde, utilizou-se distribuição unilateral, visto que neste caso, só é interessante verificar o limiar entre a situação de controle (endemia) e uma situação fora de controle (epidemia). Para os dados meteorológicos foi utilizado o bilateral, pois neste caso, é importante especificar a faixa de variação, em que os elementos meteorológicos, foram corretamente estimados para o período em estudo. Em ambos os casos, os dados utilizados neste estudo foram estimados com um nível de $95 \%$ de confiança. Os dados referentes a incidência de casos de Dengue, Meningite e Pneumonia foram coletados junto à Secretaria de Saúde do Estado da Paraíba.

As variações climáticas mensais, e por estação do ano do período estudado, foram avaliadas por análise direta das variáveis e por métodos estatísticos.

Em seguida fez-se uso da análise de regressão simples e, em seguida, a regressão linear múltipla com todos os elementos meteorológicos contidos no modelo, com a finalidade de saber o máximo que as condições meteorológicas estudadas explicam sobre a incidência das patologias estudadas. Por fim, fez-se uso do método de regressão múltipla denominada "stepwise" regression procedure, para manter no modelo apenas elementos meteorológicos que mais contribuam sobre a incidência das patologias na localidade.

A reta de mínimo quadrado que melhor se ajusta ao conjunto de pontos $\left(\mathrm{X}_{1}, \mathrm{Y}_{1}\right),\left(\mathrm{X}_{2}, \mathrm{Y}_{2}\right), \ldots .,\left(\mathrm{X}_{\mathrm{N}}, \mathrm{Y}_{\mathrm{N}}\right)$ será representada pela equação:
$\mathrm{Y}=\mathrm{a}_{0}+\mathrm{a}_{1} \mathrm{X}$

em que, as constantes $\mathrm{a}_{0}$ e $\mathrm{a}_{1}$ são denominadas mediante ao comumente encontrado na literatura Spiegel (1998).

A expressão matemática usada para a obtenção do coeficiente de correlação r, é a que segue Spiegel (1998):

$\mathrm{R}=\mathrm{S}_{\mathrm{xy}} / \mathrm{S}_{\mathrm{x}} \mathrm{S}_{\mathrm{y}}$

em que, $S_{x y}$ é a covariância amostral e $S_{x}$ e $S_{y}$ são os desvios padrões das séries de dados. A variação independente, $\mathrm{Y}$, é a doença e as variáveis dependentes, $\mathrm{X}$, os elementos meteorológicos.

Se $\mathrm{R}=0$, não há correlação entre as variáveis, $\mathrm{se} \mathrm{R}=1$, a relação é funcional e positiva, se $\mathrm{R}=-1$, a relação é funcional e negativa.Essas propriedades podem ser vistas em Griffiths (1967), Bussab (1988) e Wilks (1995).

Uma regressão múltipla, consiste de $\mathrm{n}$ observações de $\mathrm{p}$, variáveis independentes ou explicativas $\left(\mathrm{X}_{1}, \mathrm{X}_{2}, \ldots, \mathrm{X}_{\mathrm{p}}\right)$ e uma variável dependente ou resposta $(\mathrm{Y})$.

A relação entre $\mathrm{Y}$ e $\mathrm{X}_{1}, \mathrm{X}_{2}, \mathrm{X}_{3}, \ldots, \mathrm{X}_{\mathrm{p}}$ é formulada como no modelo linear

$$
\mathrm{Y}=\mathrm{B}_{0}+\mathrm{B}_{1} \mathrm{X}_{1 \mathrm{i}}+\mathrm{B}_{2} \mathrm{X}_{2 \mathrm{i}}+\ldots+\mathrm{B}_{\mathrm{p}} \mathrm{X}_{\mathrm{pi}}+\mathrm{U}_{\mathrm{i}}
$$

em que $B_{0}, B_{1}, B_{2}, \ldots, B_{p}$ são os coeficientes de regressão e $U_{i}$ são os distúrbios aleatórios, independentemente distribuídos, com média zero e variância constante $\mathrm{e} Y$ é uma função linear dos $X_{\mathrm{i}^{\prime} \mathrm{s}}$. $\mathrm{O}$ coeficiente de regressão, $\mathrm{B}_{\mathrm{i}}$, pode ser interpretado como um incremento em $\mathrm{Y}$, correspondente a um acréscimo de uma unidade em $\mathrm{X}_{\mathrm{i}}$, quando todas as outras variáveis forem mantidas constantes. Em resumo, a equação (7) é a equação de um plano no espaço $\mathrm{p}$ dimensional.

Os $\mathrm{B}_{\mathrm{i} \text { 's }}$ são estimados pela minimização da soma dos quadrados dos resíduos ou método dos mínimos quadrados (MMQ), e são obtidos resolvendo o conjunto de equações normais do plano dado por (8) ou conjunto de $n$ equações múltiplas lineares:

$\sum_{\mathrm{i}=1}^{\mathrm{N}} \mathrm{Y}_{\mathrm{i}} \mathrm{X}_{1 \mathrm{i}}=\mathrm{B}_{0} \sum_{\mathrm{i}=1}^{\mathrm{N}} \mathrm{X}_{1 \mathrm{i}}+\mathrm{B}_{1} \sum_{\mathrm{i}=1}^{\mathrm{N}} \mathrm{X}_{1 \mathrm{i}}^{2}+$

$\mathrm{B}_{2} \sum_{\mathrm{i}=1}^{\mathrm{N}} \mathrm{X}_{1 \mathrm{i}} \mathrm{X}_{2 \mathrm{i}}+\ldots+\mathrm{B}_{\mathrm{P}} \sum_{\mathrm{i}=1}^{\mathrm{N}} \mathrm{X}_{1 \mathrm{i}} \mathrm{X}_{\mathrm{Pi}}$

$\sum_{\mathrm{i}=1}^{\mathrm{N}} \mathrm{Y}_{\mathrm{i}} \mathrm{X}_{\mathrm{ni}}=\mathrm{B}_{0} \sum_{\mathrm{i}=1}^{\mathrm{N}} \mathrm{X}_{\mathrm{ni}}+\mathrm{B}_{1} \sum_{\mathrm{i}=1}^{\mathrm{N}} \mathrm{X}_{\mathrm{li}}^{2}+\mathrm{B}_{2} \sum_{\mathrm{i}=1}^{\mathrm{N}} \mathrm{X}_{1 \mathrm{i}} \mathrm{X}_{2 \mathrm{i}}+$

$\ldots+\mathrm{B} \sum_{\mathrm{i}=1}^{\mathrm{N}} \mathrm{X}_{2 \mathrm{i}}+\ldots+\mathrm{B}_{\mathrm{P}} \sum_{\mathrm{i}=1}^{\mathrm{N}} \mathrm{X}_{\mathrm{ni}} \mathrm{X}_{\mathrm{Pi}}$

Uma das vantagens, de uma função de regressão que incorpora muitas variáveis importantes, relacionadas a variável dependente (Y), é que ela permite estudar como uma dessas variáveis independentes afeta $\mathrm{Y}$, quando todas as outras variáveis são mantidas constantes. 
Após ajustar os dados observados ao modelo, a avaliação desse modelo, poderá ser efetuada pelo coeficiente de determinação $\left(\mathrm{R}^{2}\right)$, da regressão múltipla, dada por:

$$
\mathrm{R}^{2}=1-\sum_{\mathrm{i}=1}^{\mathrm{N}}\left(\mathrm{Y}_{\mathrm{obs}}-\mathrm{Y}_{\text {est }}\right)^{2} / \sum_{\mathrm{i}=1}^{\mathrm{N}}\left(\mathrm{Y}_{\mathrm{obs}}-\mathrm{Y}\right)^{2}
$$

Testar a hipótese de que não existe $\mathrm{B}_{0}=\mathrm{B}_{1}=\mathrm{B}_{2} \ldots=\mathrm{B}_{\mathrm{n}}$, é equivalente a testar a hipótese, de que não existe associação linear entre os valores das variáveis independentes e da variável dependente. Essa hipótese é testada pelo teste $\mathrm{F}$ que é dado por:

$\mathrm{F}=\mathrm{R}^{2}(\mathrm{n}-\mathrm{P}-1) /\left(1-\mathrm{R}^{2}\right) \mathrm{P}$

em que ( $\mathrm{n}-\mathrm{P}-1$ ) é o grau de liberdade, $\mathrm{n}$ é o tamanho da amostra e p é o número de parâmetros do modelo. Quando o valor de $\mathrm{F}$ calculado for maior do que o $\mathrm{F}$ tabelado, para um dado nível de significância $(1-\sigma)$, cujo valor utilizado foi 0,05 , rejeita-se a hipótese dos $\mathrm{B}_{\mathrm{i} \text { 's }}$ serem nulos e aceita-se a regressão. Essas definições podem ser vistas em Spiegel (1998), Weisberg (1980), Draper \& Smith (1981).

Utilizaram-se, para analisar os resultados obtidos neste trabalho, os seguintes critérios:

Critério para avaliar a contribuição de uma variável no modelo obtido pelo ajuste de regressão múltipla, Maia (2001), de acordo com Draper \& Smith (1981), utilizando o método de "stepwise" regression procedure: permanece no modelo obtido pelo ajuste da regressão múltipla, as variáveis independentes que melhor se ajustam ao fenômeno, não apenas pelo critério da significância estatística, mas, também com base na contribuição da cada variável ao modelo. Esta contribuição quantitativa foi estimada pelo aumento observado na soma de quadrado devido à regressão, quando da adição de cada variável no modelo. Em seguida, somam-se todos os pesos das variáveis assumindo-os iguais a $100 \%$, e fez-se a proporção percentual de cada variável. O maior peso multiplicado por 0,9 , foi o valor limite para determinar as variáveis que podem ser consideradas preditoras.

Tabela 1 - valores críticos de temperatura e umidade relativa do ar, utilizando como referência, a normal climatológica dos últimos 30 anos, da localidade em estudo, 1961 - 1990.

\section{João Pessoa}

Alta temperatura, $\mathrm{T}>26,1{ }^{\circ} \mathrm{C} ; \mathrm{T}_{\mathrm{x}}>29,3{ }^{\circ} \mathrm{C} ; \mathrm{T}_{\mathrm{n}}>21,9^{\circ} \mathrm{C}$

Baixa temperatura, $\mathrm{T}<26,1{ }^{\circ} \mathrm{C} ; \mathrm{T}_{\mathrm{x}}<29,3{ }^{\circ} \mathrm{C} ; \mathrm{T}_{\mathrm{n}}<21,9{ }^{\circ} \mathrm{C}$

Alta Umidade, UR > 76,2 (\%)

Baixa Umidade, UR $<76,2(\%)$

\section{RESULTADOS E DISCUSSÃO}

\subsection{Incidência do Dengue}

Dentro do período analisado, 1992 - 2000, nos anos de 1992 a 1995 não foi registrado nenhum caso de dengue em ambas as localidades estudadas. Isto se deveu ao fato do mosquito vetor, transmissor desse vírus, ainda não ter sido importado para a localidade de João Pessoa. Justifica-se dessa forma, o fato do período estudado para essa patologia, constar apenas dos anos de 1996 a 2000, já que o período inicial de incidência do dengue neste município ocorreu a partir de 1996.

\subsubsection{Incidência Mensal do Dengue}

A Figura 1 mostra respectivamente, o perfil do dengue no Município de João Pessoa em médias mensais/10.000 habitantes para o período de 1996 - 2000, e o comportamento das variáveis meteorológicas estudadas nesta pesquisa para este mesmo período.

A Figura 1a revelou nitidamente o perfil do dengue para João Pessoa. Os meses de maior incidência ocorrem no período de Março a Junho, sendo Maio, o mês de pico com 17,47 casos/ 10.000 habitantes. Até Maio, a temperatura ainda encontra-se elevada e o período chuvoso está acontecendo, como pode ser visto na Figura 1f. A partir de Junho, mês em que a temperatura começa a decrescer, decresce também o número de casos da doença, que prossegue decrescendo até Dezembro, mês de menor incidência com 0,57 casos/ 10.000 habitantes.

A Figura 2 mostra o número máximo de casos esperados desta doença, a partir de 2001, para o mês de Maio, é de 41,29 casos/10.000 habitantes, uma vez que, acima desse valor, atingese o estado de epidemia.

A partir da analise da incidência mensal de dengue, pode-se afirmar que há um maior favorecimento para a disseminação do vírus do dengue nos cinco primeiros meses do ano conforme afirma o Ministério da Saúde (1998) e Veronesi (1999).

Nos meses em que a temperatura começa a baixar, em seguida, percebe-se um decréscimo do número de casos incidentes. Isto sugere, a existência de uma faixa considerada ótima, que favorece a proliferação do mosquito vetor como também a transmissão do vírus causador dessa patologia, já que, quanto maior o número de mosquitos maior será o risco de contaminação. Por outro lado, as baixas temperaturas podem causar a redução do número de registros de casos, principalmente no início do período considerado frio, sugerindo que este decréscimo pode estar relacionado com a perda do poder de infecção do mosquito vetor, concordando com Patz et al.,(1996) Sabbatini, (1997), Veronesi,(1999) e Epstein (2000). 


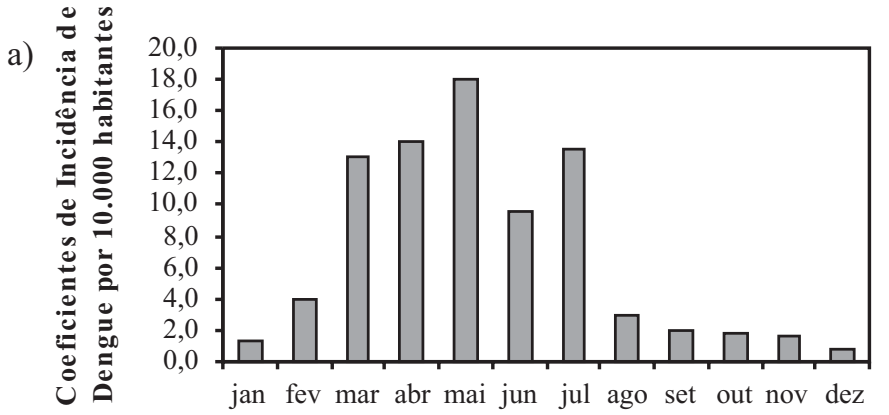

Mês

c)

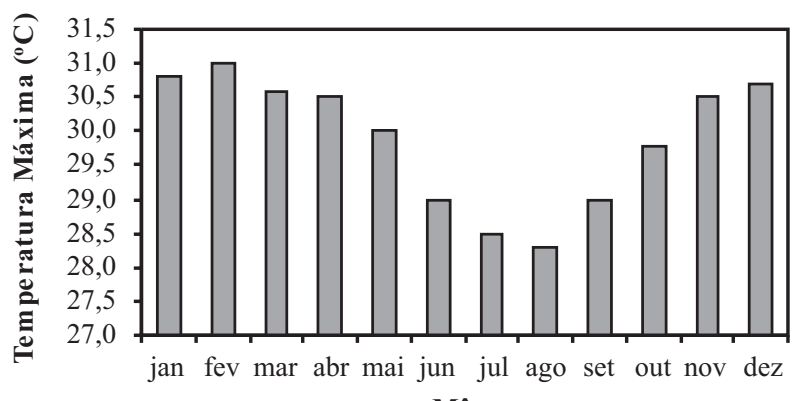

Mês

e)

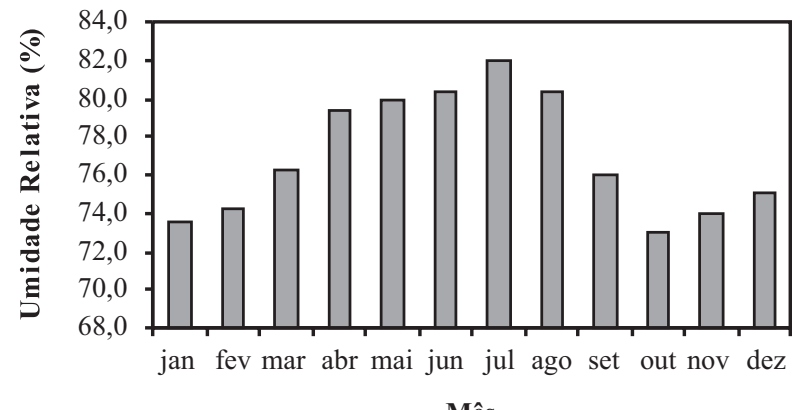

b)

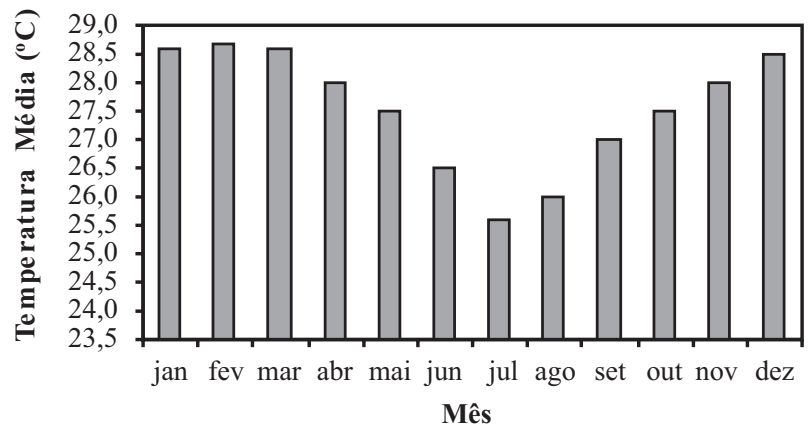

d)

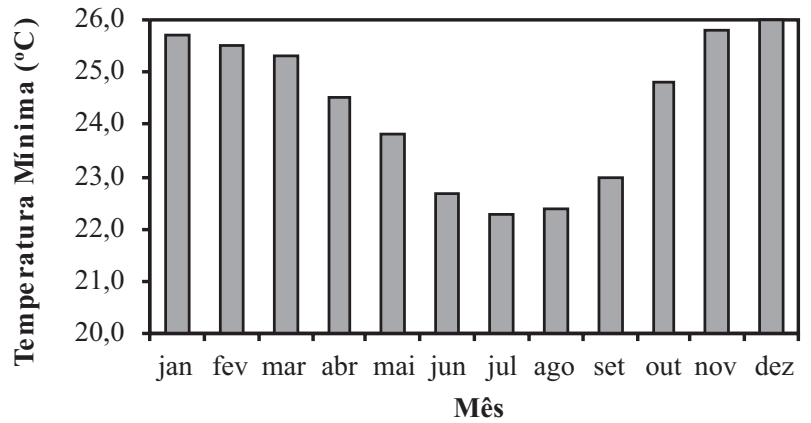

f)

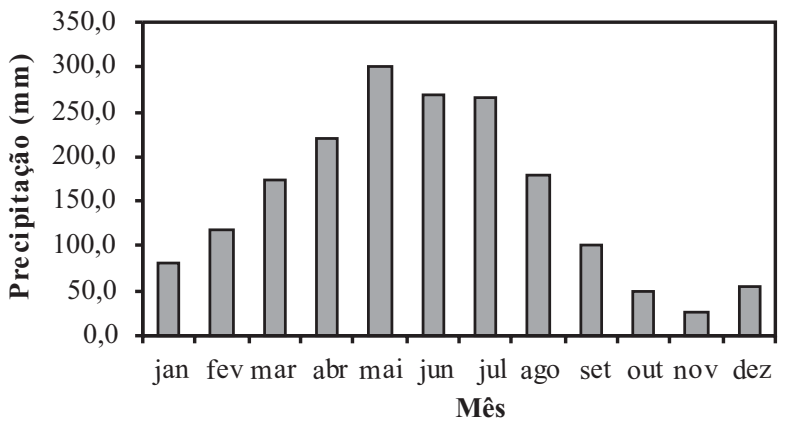

Figura 1 - Variação anual média no período estudado (1996 - 2000) do dengue (a), temperatura média (b), temperatura máxima (c), temperatura mínima (d), umidade relativa do ar (e) e precipitação (f), para a localidade de João Pessoa.

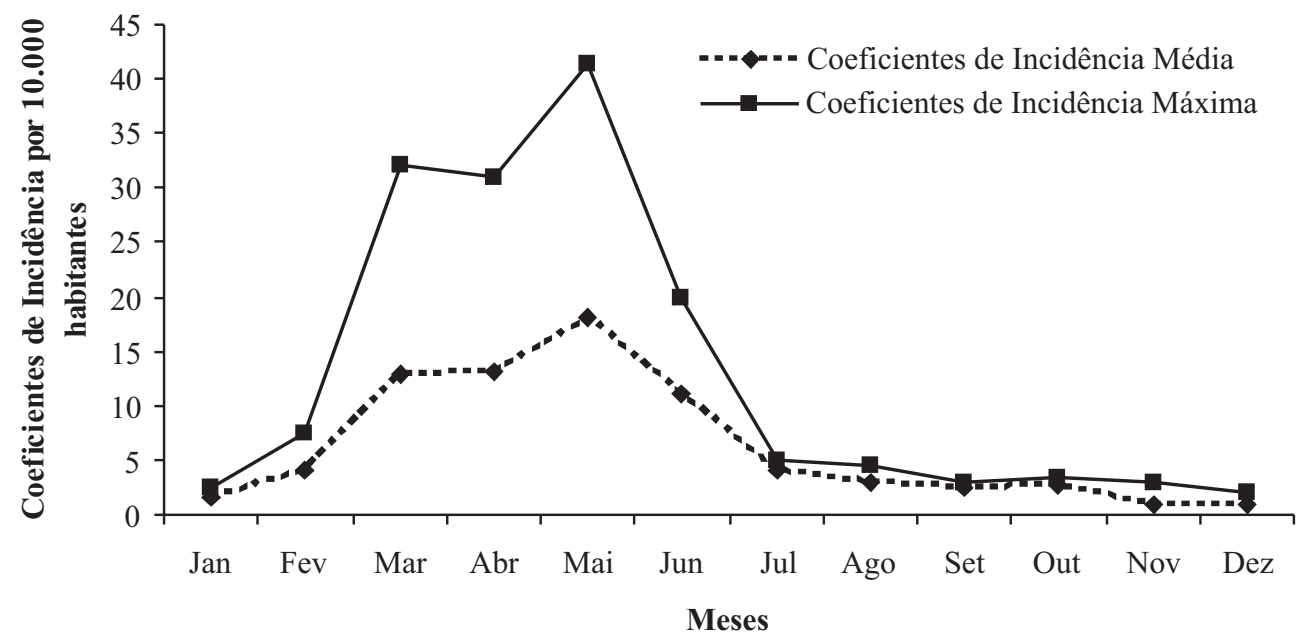

Figura 2 - Incidência média e incidência máxima esperada no período de 1996 - 2000, do dengue na localidade de João Pessoa-PB 


\subsubsection{Incidência do Dengue por Estação do Ano}

A Tabela 2, mostra a incidência de dengue, por estação do ano, em João Pessoa para o período estudado, onde se vê que, as estações do verão/outono, concentram quase a totalidade do número de casos registrados nesse período expressos em forma mensal por 10.000 habitantes. A estação de maior incidência é o outono com 13,01 casos que corresponde a $61,3 \%$ do total de casos verificados em João Pessoa. A estação em que se observa menor ocorrência do dengue é a primavera com 0,85 casos, o equivalente a $4 \%$ do total de casos observados.

Tabela 2 - Incidência do dengue, por estação do ano, em João Pessoa (1996 - 2000), em valor médio mensal por 10.000 habitantes.

\begin{tabular}{ccccc}
\hline Localidade & Verão & Outono & Inverno & Primavera \\
\hline João Pessoa & 5,36 & 13,01 & 1,99 & 0,85 \\
\hline \hline
\end{tabular}

Durante o verão, observa-se que na localidade de João Pessoa, há indícios que as condições atmosféricas favoreçam mais a proliferação e contaminação do mosquito vetor, já que, com a temperatura e umidade elevadas neste local, durante esta estação, propiciam condições adequadas para aumentar o poder de infecção da fêmea, e assim, conseguir espalhar o vírus, com maior rapidez, concordando com Moraes et al. (1996).

No outono nesta localidade, período de intensificação das chuvas, esse favorecimento atmosférico parece aumentar ainda mais que na estação anterior, principalmente, até meados do período. Em seguida, reduz-se bastante, o poder do mosquito de infectar e transmitir o vírus, isto é, de completar o seu ciclo extrínseco. Isto sugere, que a perda de tal poder esteja condicionado as temperaturas mínimas, já que o mosquito requer além de chuvas, temperaturas elevadas para procriar e infectar o ser humano.

Durante o inverno, há evidências de que as condições atmosféricas nesta estação sejam adversas para o mosquito vetor, inibindo a proliferação e a contaminação do mesmo, pois as baixas temperaturas, principalmente a temperatura mínima, impedem que seu ciclo extrínseco se complete.

Na primavera, apesar da temperatura já está elevada, a baixa umidade relativa do ar, parece inibir a ação do mosquito vetor, sugerindo que, nesse período, as condições atmosféricas, também sejam desfavoráveis ao Aedes aegypti.

\subsection{Incidência de Meningite}

\subsubsection{Incidência Mensal de Meningite}

A Figura 3 mostra respectivamente, o perfil de meningite no Município de João Pessoa em médias mensais/10.000 habitantes para o período de 1992 - 2000, e também o comportamento das variáveis meteorológicas estudadas na pesquisa.

A Figura 3a apresenta os meses de picos de incidência da doença em João Pessoa, Março e período de Julho a Outubro. Em Março, a alta temperatura, e em Julho e Agosto, a baixa temperatura e a alta umidade relativa do ar, juntamente com a precipitação mais intensa, podem estar relacionados ao favorecimento do aumento desta enfermidade, concordando com o que foi observado por Gama (1995). Quanto aos meses de Setembro e Outubro, a baixa umidade relativa do ar e o aumento da temperatura parecem não contribuir com o aumento da incidência desta enfermidade neste local. Os meses de Março e Julho são considerados de maior incidência com 0,38 casos/ 10.000 habitantes nesta localidade.

Na Figura 4 observa-se a faixa de incidência normal esperada da meningite, para o município de João Pessoa. Como se vê, o coeficiente de incidência máximo esperado para o mês de Fevereiro, a partir de 2001, é de cerca de 0,48 casos $/ 10.000$ habitantes. Isto, se a estrutura epidemiológica permanecer inalterada.

Como se pode vê, uma justificativa para o comportamento da incidência da meningite em João Pessoa, é que a mesma trata-se de uma enfermidade universal e ocorre durante todo o ano, principalmente nos meses menos quentes, pois além de haver melhores condições para o germe se proliferar no meio ambiente, a doença esta inter-relacionada com outras doenças, conforme ressaltou o Ministério da Saúde (1975).

\subsubsection{Incidência de Meningite por Estação do Ano}

A Tabela 3 apresenta a incidência de meningite por estação do ano, em João Pessoa, em médias mensais para o período 1992 - 2000.

Nesta localidade, a estação que concentra maior número de casos notificados é o inverno, com 0,37 casos por mês em 10.000 habitantes. Isto equivale a aproximadamente $28,5 \%$ do total de casos verificados no município.

No verão a alta temperatura, a baixa umidade relativa do ar, e o início do período chuvoso, no final da estação, parecem contribuir no aumento de casos de meningite, em João Pessoa no final deste período. No outono, à medida que a temperatura diminui e, a umidade e a precipitação aumentam, parece haver um favorecimento para o aumento de casos desta enfermidade neste local. Há evidências de que no inverno, a baixa temperatura e a alta umidade relativa do ar contribuam com o aumento do número de casos de meningite neste município.

É possível que o aumento da temperatura e a redução da umidade relativa do ar, contribuam para reduzir o número de ocorrências desta enfermidade durante a primavera. 
a)

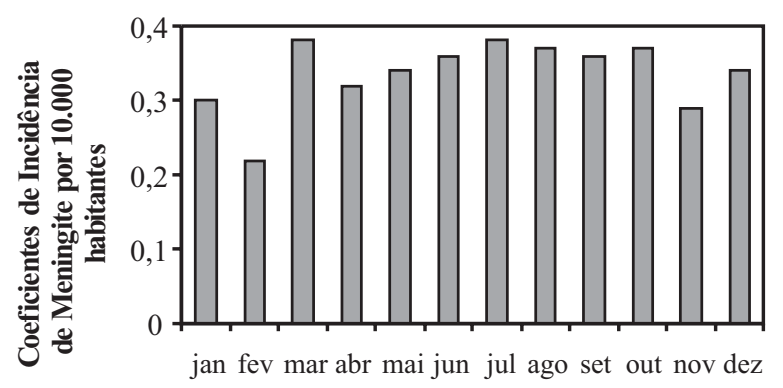

Mês

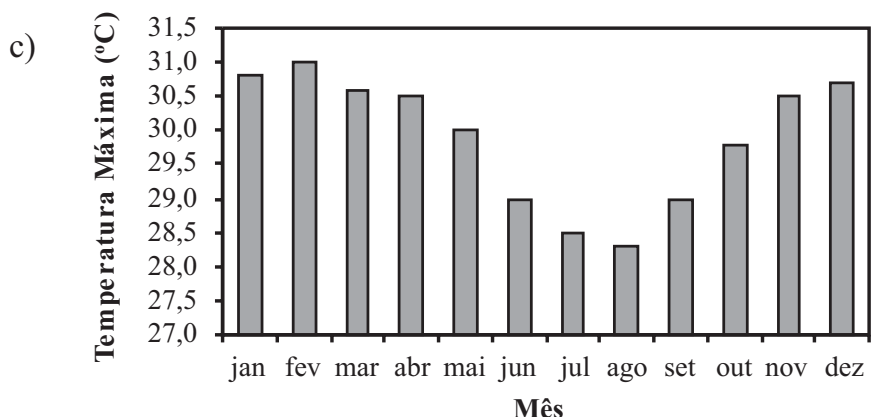

e)

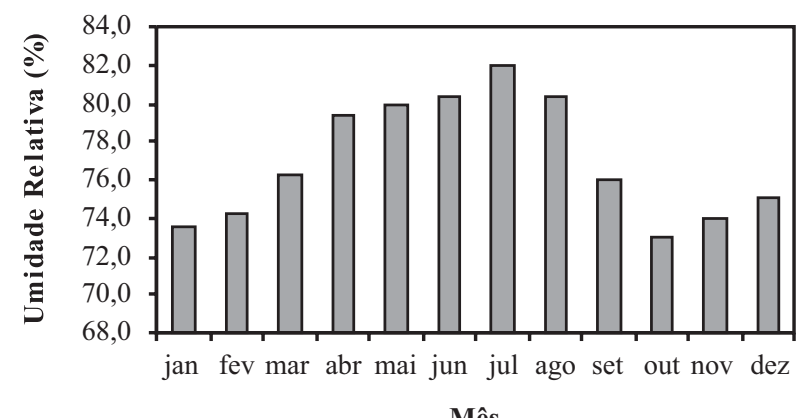

b)

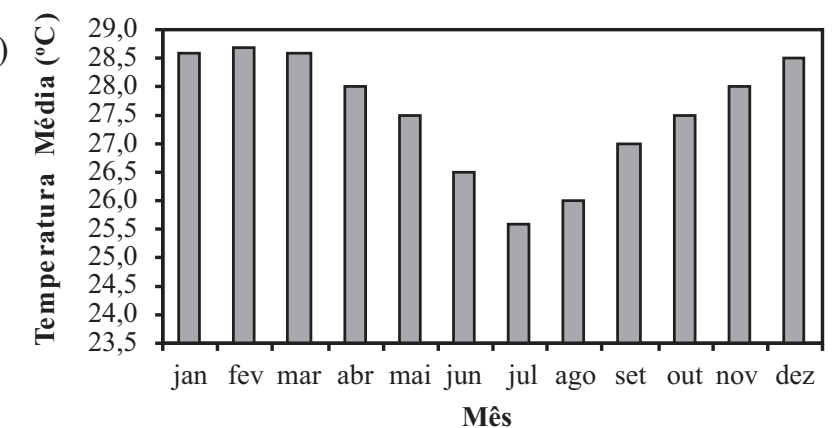

d)
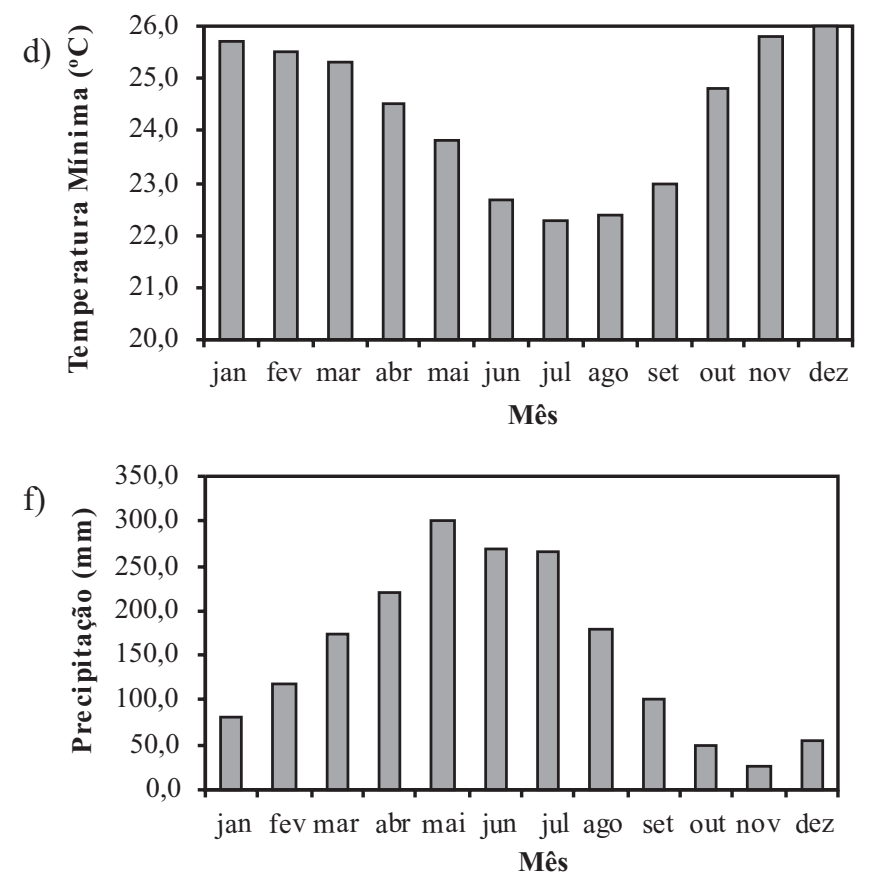

Figura 3 - Variação média anual no período estudado (1992 - 2000) de meningite (a), temperatura média (b), temperatura máxima (c), temperatura mínima (d), umidade relativa do ar (e) e precipitação (f), para a localidade de João Pessoa.

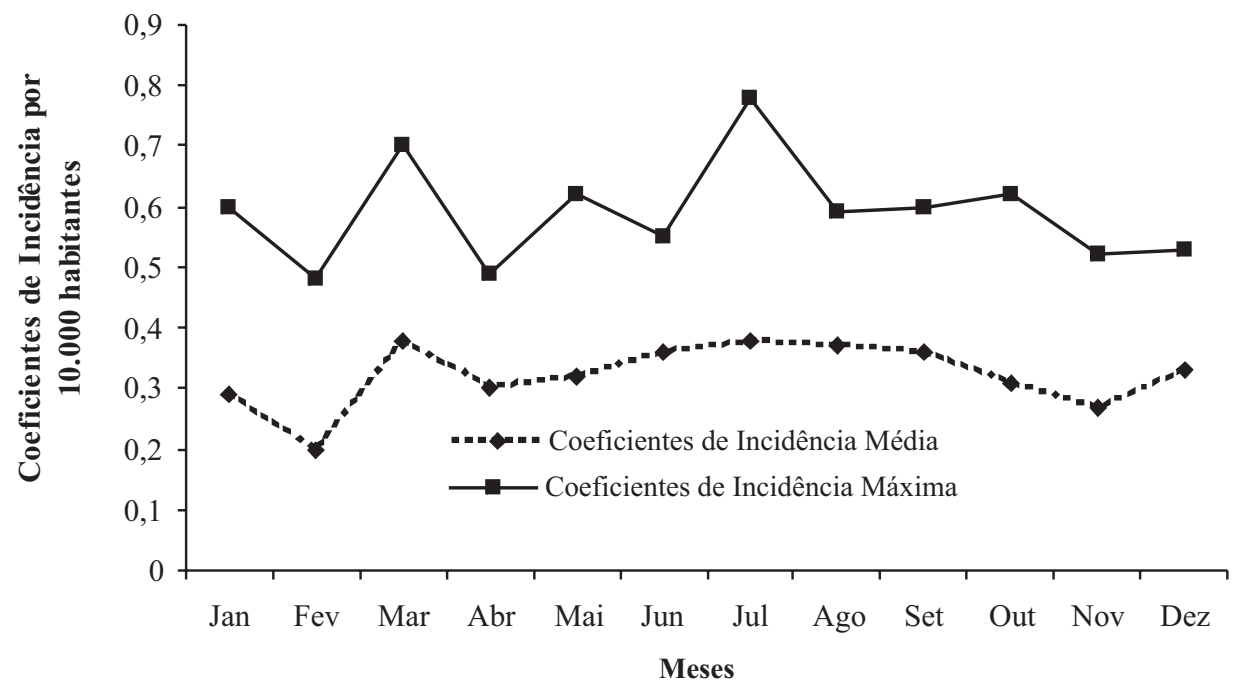

Figura 4 - Incidência média e incidência máxima esperada no período de 1992 - 2000, da meningite na localidade de João Pessoa-PB. 
Tabela 3 - Incidência de meningite, por estação do ano, em João Pessoa, 1992 - 2000, em valor médio mensal por 10.000 habitantes.

\begin{tabular}{ccccc}
\hline \hline Localidade & Verão & Outono & Inverno & Primavera \\
\hline \hline João Pessoa & 0,29 & 0,32 & 0,37 & 0,32 \\
\hline
\end{tabular}

A época de ocorrência mais freqüente desta enfermidade depende do agente etiológico, bactérias, vírus, fungos e outros microorganismos patogênicos e do tipo de meningite. Uma justificativa para o pico registrado na localidade de estudo no mês de Março poderia ser a ocorrência de meningites virais, que ocorrem mais freqüentemente no final do verão e começo do outono. E para o pico de Julho, mês considerado menos quente, poderia ser a ocorrência de meningite espinhal, prolongando-se também no outono e primavera.

\subsection{Incidência de Pneumonia}

\subsubsection{Incidência Mensal de Pneumonia}

Na Figura 5, observa-se respectivamente, o perfil de pneumonia no Município de João Pessoa em médias mensais/10.000 habitantes para o período de 1992 - 2000, e o comportamento das variáveis meteorológicas estudadas nesta pesquisa para este mesmo período.

A Figura 5a, mostra que em João Pessoa, nos meses em que a temperatura média $(\mathrm{T})$, temperatura máxima $\left(\mathrm{T}_{\mathrm{x}}\right)$ e temperatura mínima $\left(\mathrm{T}_{\mathrm{n}}\right)$ decrescem e, (UR) umidade relativa do ar e (P) precipitação aumentam significativamente, logo se percebe um aumento na ocorrência de pneumonia neste local. Sendo os meses de Abril a Julho, o período de maior incidência, com o mês de Junho registrando o maior número de ocorrências, com 9,55 casos/ 10.000 habitantes.

A Figura 6 a seguir representa a faixa de incidência normal esperada, de pneumonia, para o município de João Pessoa. Como se vê o coeficiente de incidência máximo esperado para o mês de Junho, a partir de 2001, é de cerca de 15,82 casos / 10.000 habitantes.

\subsubsection{Incidência de Pneumonia por Estação do Ano}

A Tabela 4 apresenta a incidência da pneumonia, por estação do ano, em João Pessoa para o período estudado, em valores médios mensais por 10.000 habitantes. Nela, se pode vê que as estações do outono / inverno concentram um pouco mais da metade do número de casos registrados nesse período, com cerca de $53 \%$ do total dos casos. A estação de maior incidência é o outono, com 9,05 casos que corresponde a $29 \%$ do total verificado em João Pessoa. A estação em que se observa menor ocorrência de pneumonia é o verão, com 7,3 casos mensais em João Pessoa, que é equivalente, respectivamente, a cerca de $23,5 \%$ do total dos casos observados.

Tabela 4 - Incidência de Pneumonia, por estação do ano, em João Pessoa, 1992 - 2000, em valor médio mensal por 10.000 habitantes.

\begin{tabular}{ccccc}
\hline Localidade & Verão & Outono & Inverno & Primavera \\
\hline \hline João Pessoa & 7,29 & 9,05 & 7,52 & 7,36 \\
\hline \hline
\end{tabular}

Uma justificativa para os valores apresentados acima, é que, no verão a alta temperatura, a baixa umidade relativa do ar, e o início do período chuvoso, no final da estação, coincidem com o aumento dos casos de pneumonia, em João Pessoa. No outono, à medida que as temperaturas, média, máxima e mínima estão baixando e, a umidade relativa do ar e a precipitação aumentando, o número de casos registrados desta doença começam a aumentar, e prossegue aumentando até o final desta estação, concordando com informações do Hospital das Clínicas da Faculdade de Medicina de São Paulo (2001), no que se refere ao pico de incidência desta enfermidade, durante a estação chuvosa, neste local. No inverno, é possível que o aumento da temperatura juntamente, com a redução da umidade e da precipitação em meados deste período, favoreçam o decréscimo do número de casos notificados nesta estação.

A alta temperatura, a baixa umidade relativa do ar e a escassez de chuvas, durante a primavera, parecem contribuir discretamente na redução do número de casos no decorrer desta estação; porém, observa-se, entretanto, um pequeno aumento na incidência da doença, no início deste período.

Uma limitação deste trabalho está na base de dados, pois não se dispõe de uma série de anos anteriores a 1992, não sendo possível comparar a endemicidade destas patologias, para melhor investigar qual o fator climático ou não, que na década em estudo, pode ser associado ou não, a alterações na estrutura epidemiológica de uma doença. Ressaltando que, é necessário no mínimo 10 anos de dados para se delinear o perfil de incidência de uma determinada doença, daí o caráter experimental deste trabalho.

Vale salientar ainda que, não existem até o momento provas científicas da influência climática sobre o organismo humano e que a Academia Nacional de Saúde dos EEUU recomendam cautela nas conclusões das pesquisas relacionando clima e saúde. 
a)

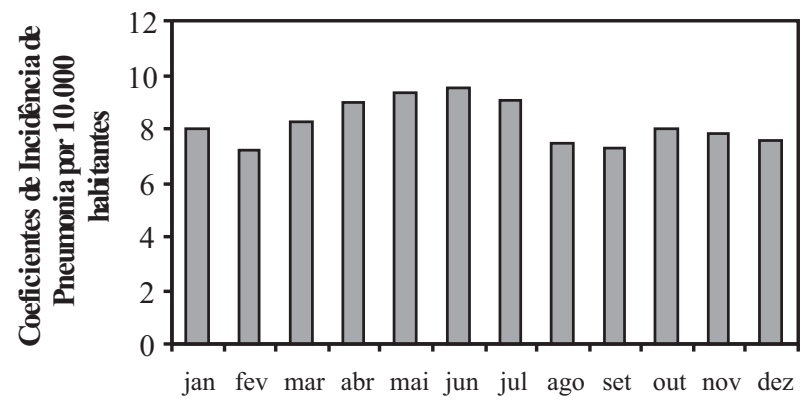

Mês

c)

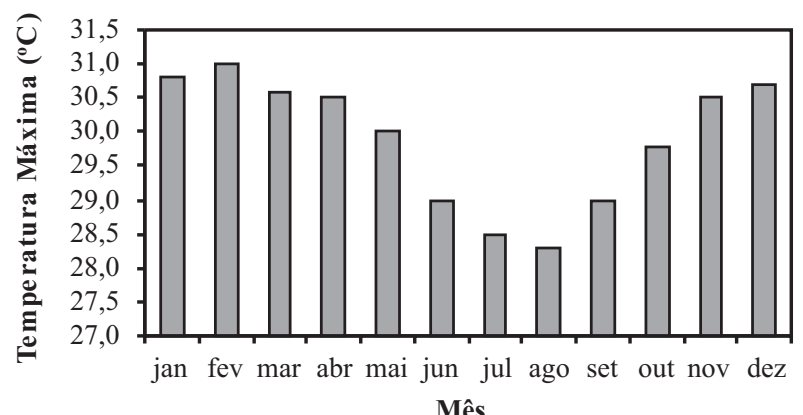

e)

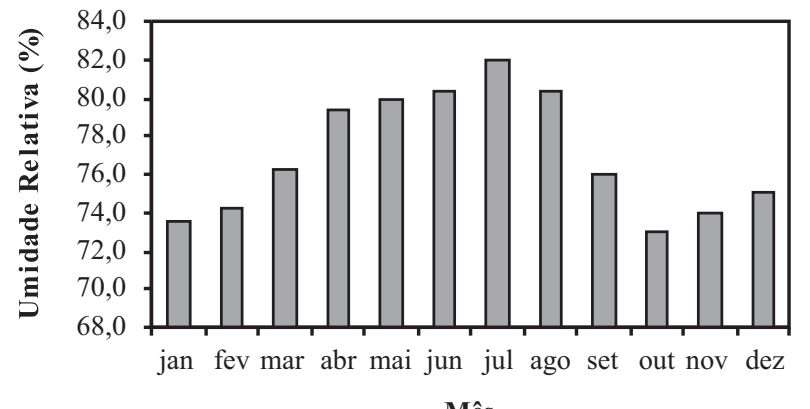

b)

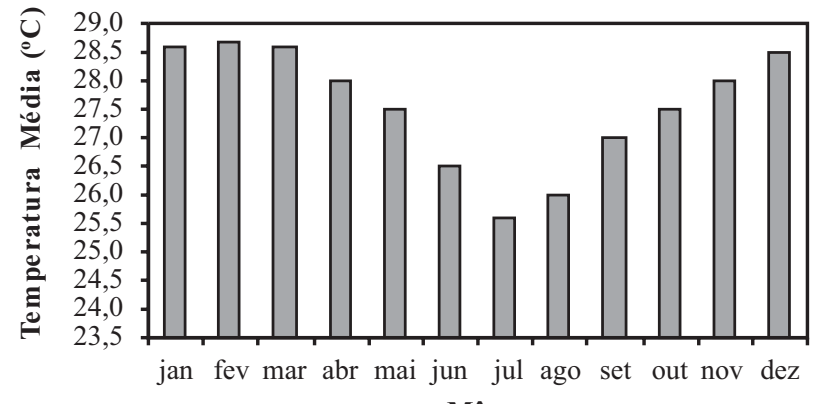

Mês

d)

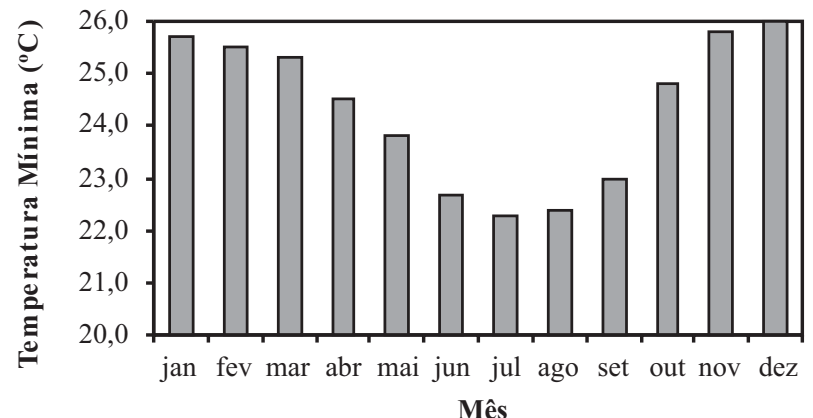

f)

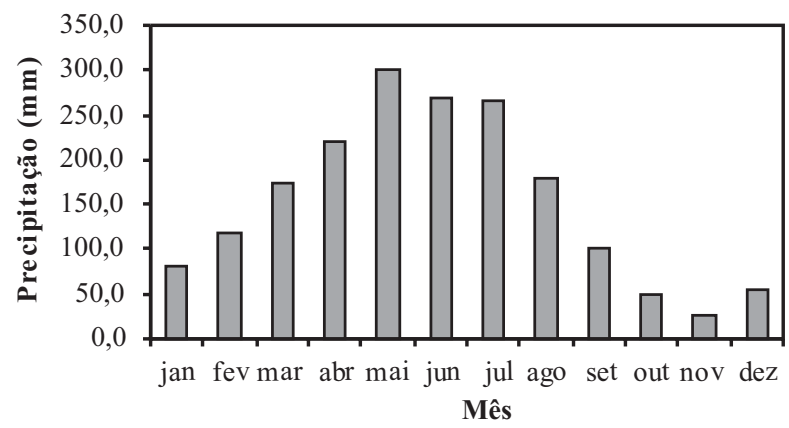

Figura 5 - Variação anual média no período estudado (1992 - 2000) de pneumonia (a), temperatura média (b), temperatura máxima (c), temperatura mínima (d), umidade relativa do ar (e) e precipitação (f), para a localidade de João Pessoa.

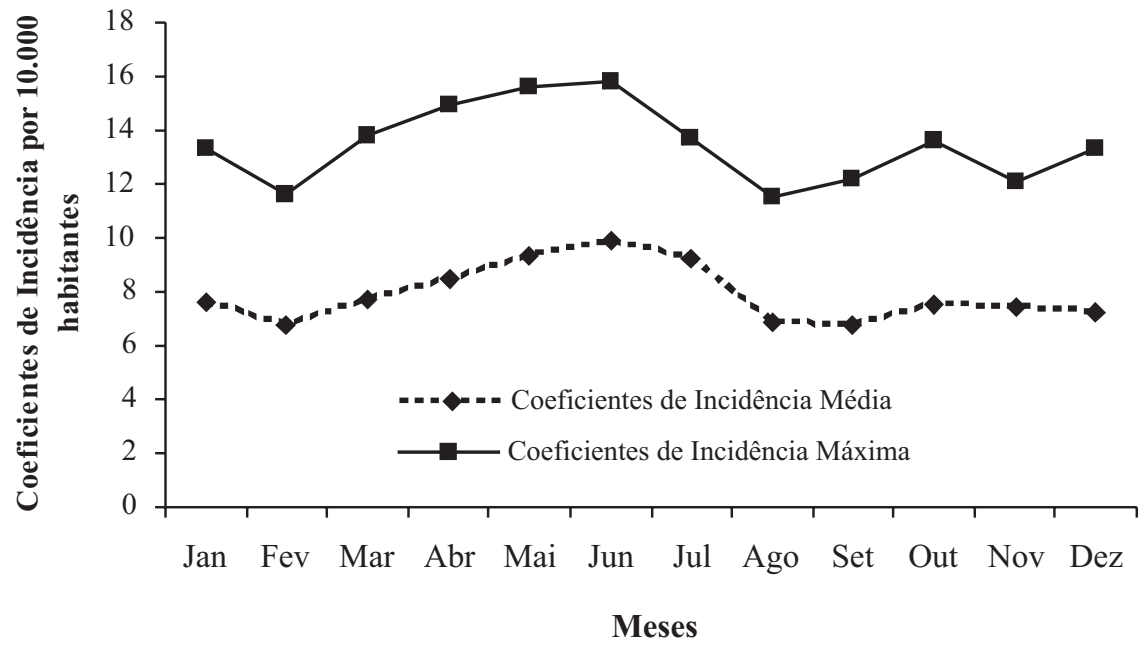

Figura 6 - Incidência média e incidência máxima esperada no período de 1992 - 2000, de pneumonia na localidade de João Pessoa-PB. 


\section{CONCLUSÕES}

Houve influência dos elementos meteorológicos sobre a incidência de dengue em João Pessoa, onde o aumento de $1^{\circ} \mathrm{C}$, na temperatura máxima mensal, provoca aumento de aproximadamente 8 casos/mês de incidência de dengue, ao passo que aproximadamente cada $35 \mathrm{~mm}$ de aumento na precipitação média mensal, provoca um aumento de 1 caso/mês, podendo estas variáveis serem consideradas como preditoras para o número de casos de dengue neste local;

Houve influência dos elementos meteorológicos sobre a incidência de pneumonia em João Pessoa, sendo que a cada $1^{\circ} \mathrm{C}$ de diminuição na temperatura média mensal, encontrou-se um aumento de aproximadamente 1 caso mês/10.000 habitantes. Este elemento meteorológico pode ser considerado como preditor para o número de casos de pneumonia nesta localidade.

As condições atmosféricas de João Pessoa favorecem a ação do mosquito vetor de transmitir o vírus da dengue, propiciando sua proliferação mais rápida.

A influência mensal e sazonal da temperatura do ar é muito significativa sobre a incidência de dengue, meningite e pneumonia em João Pessoa.

Os elementos meteorológicos, considerados na pesquisa, contribuíram sobre a incidência do dengue, meningite e pneumonia em João Pessoa na faixa de $34 \%$, justificando que além das condições meteorológicas, existem outras causadas por condições nutricionais, sociais, e de defesa imunológica do organismo humano.

\section{REFERÊNCIAS BIBLIOGRÁFICAS}

BRASIL. Ministério da Saúde. Dengue. Brasília: Fundação Nacional de Saúde, 1998.

BUSSAB, W.O. Analise de variância e de regressão; uma introdução. 2 ed. São Paulo: Atual, 1998.

BRASIL. Ministério da Saúde.Fundação Nacional de Saúde. Doença Meningocócica. Vigilância Epidemiológica e Controle, 1975. 48p.

DRAPPER, N.R.; SMITH, H. Applied regression analyses. 2.ed. New York: John Wiley \& Sons, 1981. 709p.
EPSTEIN, P. Is Global warming harmful to healt? Scientific American, v. 283, p. 50-57, 2000.

GAMA, S.G.N. Doenças Meningocócica e sua evolução no município do Rio de Janeiro (1976- 1994). Rio de Janeiro, 1995. x, 195p.

GRIFFITS, J.C. Scientific method in analysis of sediments. New York: Mc Graw-Hill, 508p, 1967.

MAIA, C. E.; MORAIS, E. R. C.; OLIVEIRA, M. Classificação da composição iônica da água de irrigação usando regressão linear múltipla. Revista Brasileira de Engenharia Agrícola e Ambiental, v. 5, n. 1, p. 55-59, 2001.

MORAES, J.G.M. et al. Dengue: manual de orientações. Secretaria de Saúde do Estado de Pernambuco/Comissão Estadual de Controle do Dengue. Recife, 1996. 23p.

PATZ, J. et al. Global climate change and emerging infectious diseases. JAMA, v. 275, p. 217-223, 1996.

ROUQUAYROL, M.Z. Epidemiologia e saúde. 4. ed. Rio de Janeiro: MEDSI, 1994. 540p.

SABBATINI, R. M. E. Aquecimento global e saúde. Correio Popular. Campinas - SP, 3/10/97. Disponível em http:// www.cpopular.com.br, Acesso em: 27 de Fev. de 2002.

SÃO PAULO. Hospital das Clínicas da Faculdade de Medicina. Infecção das Vias Aéreas Superiores (IVAS) no paciente idoso, Dez. 2001. Disponível em http://www.geocities.com/ geriatriahc/ivasnoidoso.htm. Acesso em: 2 de Jun. de 2002.

SPIEGEL, M.R. Estatística. São Paulo - SP: Mc Graw-Hill, 1998. 580p.

VERONESI, R.;FOCACIA, R. Tratado de infectologia. São Paulo: Atheneu, v.1, 1996. 962p.

WEISBERG, S. Applied linear regression. New York: John Wiley and Sons, 1980.

WILKS, D.S. Estatistical methods in the atmosferic sciences. Academic Press, San Diego, Califórnia, 1995. 476p. 